\section{Jejunal post-polypectomy syndrome}

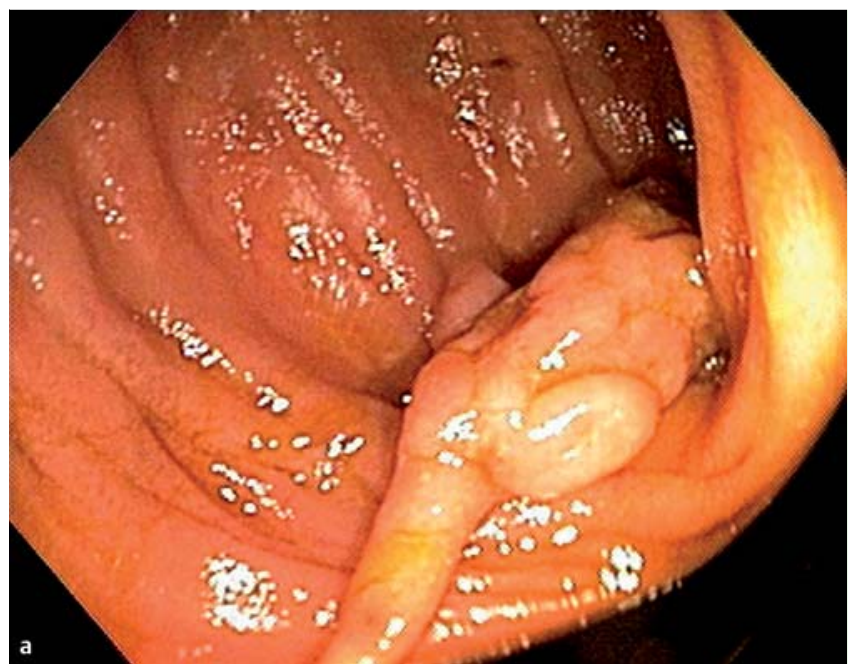

Fig. 1 a Pedunculated polyp in the proximal jejunum of a 65-year-old woman with a longstanding history of obscure gastrointestinal bleeding and anemia who was found to have active bleeding in the jejunum on capsule endoscopy. $\mathbf{b}$ The excised polyp.

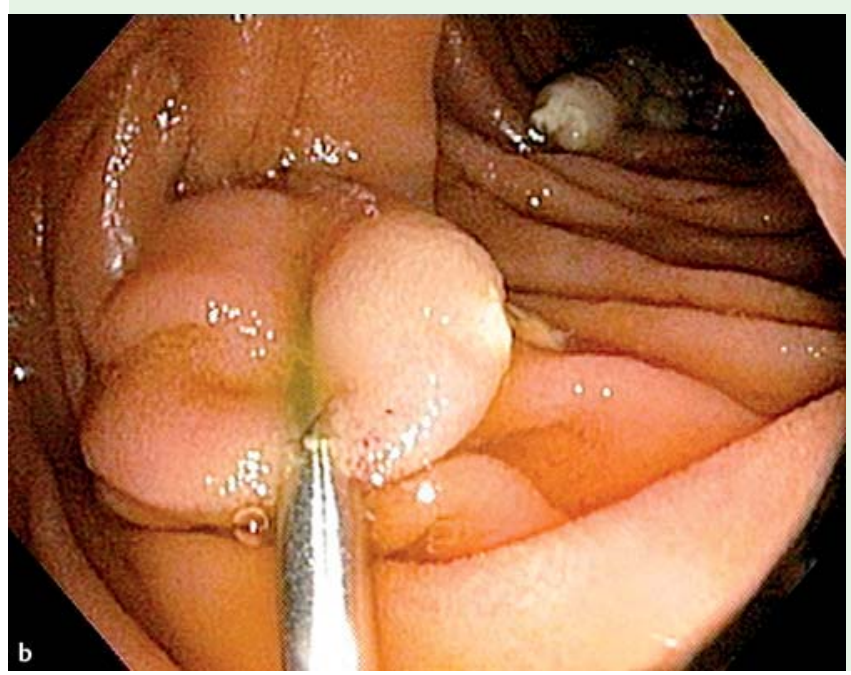

puted tomography scan showed normal findings and ruled out possible perforation or pancreatitis secondary to the procedure. Thus, a diagnosis of post-polypectomy syndrome was made. The patient was started on intravenous broad-spectrum antibiotics along with intravenous fluids and bowel rest. Her symptoms improved and she was discharged. The excised polyp was found to be a PeutzJegher polyp on pathologic examination. Post-polypectomy syndrome is a wellestablished complication after colonoscopy in which a polyp is removed by electrocoagulation. The syndrome is characterized by the development of abdominal pain, fever, leukocytosis, and peritoneal symptoms following the procedure [1]. It is thought that syndrome occurs due to extension of a transmural burn past the mucosa into the muscularis mucosa and serosa, leading to peritoneal inflammation in the absence of overt bowel perforation [2]. Risk factors that increase the likelihood of developing post-polypectomy syndrome include hypertension, large size of polyp, and nonpolypoid configuration of lesions [1]. Treatment of postpolypectomy syndrome generally consists of antibiotics and supportive care, which includes bowel rest, intravenous fluids, and slow advancement of diet [1]. Patients generally recover well with this treatment and do not display long-term stigmata. Despite extensive literature on colonic post-polypectomy syndrome, the occurrence of this condition in the small intestine has not yet been described in great detail. However, as double-balloon enteroscopy becomes more widely used for small-bowel polyp removal, its incidence will probably increase.

In conclusion, small-bowel post-polypectomy syndrome should be suspected in patients presenting with abdominal pain, fever, and leukocytosis shortly after smallintestinal polyp removal. The management and treatment should consist of early recognition of the syndrome by ruling out other causes of the symptoms along with antibiotics and supportive care, including bowel rest.

\section{Endoscopy_UCTN_Code_CPL_1AI_2AD}

Competing interests: None 


\section{Abhishek Bhandari ${ }^{1}$, Denise Kalmaz ${ }^{2}$}

${ }^{1}$ University of California at San Diego, Department of Internal Medicine, San Diego, California, United States of America

${ }^{2}$ University of California at San Diego, Department of Gastroenterology, San Diego, California, United States of America

\section{References}

1 Cha J, Lim K, Lee $S$ et al. Clinical outcomes and risk factors of post-polypectomy coagulation syndrome: a multicenter, retrospective, case-control study. Endoscopy 2013; 45: $202-207$

2 Waye J. Management of complications of colonoscopic polypectomy. Gastroenterologist 1993; 2: $158-164$

\section{Bibliography}

DOI http://dx.doi.org/

10.1055/s-0033-1344955

Endoscopy 2014; 46: E133-E134

(c) Georg Thieme Verlag KG

Stuttgart · New York

ISSN 0013-726X

\section{Corresponding author}

Abhishek Bhandari

200 W. Arbor Dr

Department of Internal Medicine

San Diego

CA 92103

USA

abbhandari@ucsd.edu 